\title{
Diagnóstico das condições de trabalho, saúde e indicadores do estilo de vida de trabalhadores do transporte coletivo da cidade de Pelotas - RS
}

Rev Bras Ativ Fis Saúde p. 347-358

DOI: http://dx.doi.org/10.12820/2317-

1634.2012v17n5p347

1 Programa de Pós-Graduação em Educação Física, Universidade Federal de Pelotas, RS, Brasil

2 GEEAF - Grupo de Estudos em Epidemiologia da Atividade Física.

\section{Diagnosis of working conditions, health and lifestyle of workers from the collective transport of Pelotas - RS}

Alvaro Braga de Moura Neto

Marcelo Cozzensa da Silva ${ }^{1,2}$

\section{Resumo}

Motoristas e cobradores de ônibus formam um grupo de risco para determinados problemas de saúde em função de características ocupacionais, como a exposição a fatores no ambiente de trabalho e na atividade executada. O objetivo do estudo foi descrever as condições de trabalho, saúde e indicadores de vida dos trabalhadores do transporte coletivo urbano da cidade de Pelotas-RS. Uma amostra de 227 motoristas e cobradores respondeu a um questionário incluindo questões sociodemográficas, econômicas, comportamentais, nutricional, de saúde e trabalho. As médias de idade e escolaridade foram respectivamente de $36,3(\mathrm{dp}=11,5)$ e $9,2(\mathrm{dp}=2,5)$ anos. Quase a totalidade dos pesquisados era do sexo masculino, 77,5\% era de cor branca, $64,3 \%$ era casado/vivia com companheiro. Quanto à autopercepção de saúde, 14,7\% dos entrevistados relatou sua saúde como regular/ruim, mais de 20,0\% dos entrevistados admitiram fumar atualmente. Quase 40\% dos trabalhadores não atingiram o mínimo de atividade física recomendada para promoção da saúde e 3/4 foram classificados nas categorias de sobrepeso/obesidade pelo Índice de Massa Corporal (IMC). As maiores prevalências de dor foram encontradas nas regiões lombar, torácica, pescoço e ombros e 9,0\% dos entrevistados apresentaram transtornos psiquiátricos menores (TPM). Motoristas e cobradores apresentam prevalências preocupantes de fatores de risco para doenças crônicas. A adequação das rotinas de trabalho e a adoção de hábitos saudáveis são pontos fundamentais para melhora da qualidade de vida e trabalho dessa categoria ocupacional.

\section{Palavras-chave}

Saúde ocupacional; Trabalho; Atividade motora; Doenças crônicas; Epidemiologia.

\begin{abstract}
Bus drivers and conductors form a group at risk for certain health problems due to occupational characteristics, as exposure in the workplace and the activity performed. The aim of this study was to describe working and health conditions and lifestyle of urban public transport workers of Pelotas - RS. A sample of 227 drivers and collectors answered a questionnaire including sociodemographic, economic, behavioral, nutritional, health and work questions. The mean age and education were respectively 36.3 (dp= $11.5)$ and $9.2(d p=2.5)$ years. Almost all the respondents were male, $77.5 \%$ were white, $64.3 \%$ were married / living with partner. As for the self perception of health, $14.7 \%$ of respondents reported their health as fair/poor, more than $20.0 \%$ of respondents admitted smoking actually. Almost $40 \%$ of workers did not reach the minimum physical activity recommended for health promotion and 3/4 were classified as overweight / obese by BMI. The highest prevalence of pain were found in lower back, chest, neck and shoulders and $9.0 \%$ of respondents had minor psychiatric disorders. The adequacy of the work routines and healthy habits are important to improve quality of life and work of this occupational category.
\end{abstract}

\section{Keywords}

Occupational Health; Labor; Motor Activity; Chronic Diseases; Epidemiology. 


\section{INTRODUÇÃO}

O transporte público é parte essencial da organização e funcionamento dos centros urbanos. Além de permitir o deslocamento em conjunto de um grande número de pessoas para diversas partes da cidade, contribui na redução do número de carros circulantes ${ }^{1}$.

De acordo com Costa ${ }^{2}$, os trabalhadores do transporte coletivo urbano constituem uma categoria profissional extremamente importante, principalmente nas sociedades mais urbanizadas, não só por formarem um contingente numeroso de trabalhadores, expostos à condições de trabalho bastante particulares, mas também, pela responsabilidade coletiva de sua atividade. No Brasil, mais de $50 \%$ da população das capitais e $36 \%$ dos pequenos e médios centros urbanos se utilizam do ônibus como meio de transporte ${ }^{3}$. Apesar dessa diferença, em geral, o instrumento e a rotina de trabalho parecem não diferir entre esses trabalhadores.

A atividade do motorista de ônibus exige atenção constante, precisão na realização das ações, autocontrole, reflexo rápido, análise e interpretação das informações fornecidas pelos equipamentos dos veículos. Dessa forma, os sistemas visual e auditivo, a percepção, a coordenação dos movimentos e o raciocínio rápido para manipular os mecanismos e equipamentos do veículo são constantemente solicitados e devem ser percebidos, analisados e respondidos em fração de segundos ${ }^{2}$.

Picoloto e Silveira ${ }^{4}$ revelam que motoristas e cobradores de ônibus formam um grupo de risco para determinados problemas de saúde em função de características ocupacionais, especialmente algumas cargas relativas ao ambiente de trabalho e à atividade que executam. Movimentos repetitivos, posição viciosa, vibração de corpo inteiro, turno alternado de trabalho e violência urbana são algumas das cargas ocupacionais a que estão expostos.

Estudo de revisão realizado por Winkleby et al. ${ }^{5}$ concluiu que motoristas de ônibus apresentam maiores taxas de mortalidade, morbidade e absenteísmo que trabalhadores de várias outras profissões, sendo o risco particularmente maior para problemas cardiovasculares, gastrointestinais e musculoesqueléticos. Tal achado pode estar relacionado ao estilo de vida e trabalho dessa população o que reforça a necessidade e importância de pesquisas envolvendo tais temáticas nesse grupo de trabalhadores. O objetivo do presente estudo foi descrever as condições de saúde, trabalho e indicadores de estilo de vida dos trabalhadores do transporte coletivo urbano de Pelotas, sul do Brasil.

\section{MÉTODOS}

Realizou-se um estudo epidemiológico de natureza descritiva, sobre as condições de saúde, trabalho e indicadores do estilo de vida dos trabalhadores do transporte coletivo urbano da cidade de Pelotas/RS. A cidade está localizada no extremo sul do estado, possui cerca de 340.000 habitantes e uma densidade demográfica de 203,89 hab/ $\mathrm{km}^{2}$ segundo o Instituto Brasileiro de Geografia e Estatística (IBGE). Existem seis empresas que prestam serviço de transporte coletivo urbano ao município, com uma frota com idade aproximada de dez anos de uso.

O universo estimado de trabalhadores do transporte coletivo no município foi de 650 indivíduos. O cálculo de tamanho amostral considerou uma prevalência estimada de 40,0\% (atividade física nos domínios de lazer + deslocamento), com margem de erro aceitável de 5,0 pontos percentuais e nível de confiança de 95\%, 
o qual, acrescendo $10 \%$ para perdas e recusas, resultou em um tamanho amostral de 233 indivíduos.

A logística do trabalho iniciou com o contato junto às empresas que atuam no transporte coletivo urbano na cidade de Pelotas para a apresentação do estudo aos gestores e obtenção da autorização para realizar a pesquisa. Nesses encontros foram solicitadas as listagens dos cobradores e motoristas de cada empresa, bem como informações relativas aos endereços de final de linha ou terminais de ônibus, horários dos itinerários, além do nome e telefone de contato dos respectivos fiscais.

Com a listagem dos trabalhadores de todas as empresas, as mesmas foram estratificadas segundo o tamanho (menos de 200 funcionários e 200 funcionários ou mais) e categorias de trabalho. A partir disso foi realizada uma amostragem aleatória proporcional ao tamanho das empresas (número de funcionários) e as categorias de trabalho (motoristas e cobradores).

A coleta de dados foi realizada no período de outubro de 2011 a fevereiro de 2012 por nove entrevistadores universitários previamente selecionados que passaram por um treinamento teórico/prático de 20 horas. Para garantir a padronização na coleta, foi utilizado um manual de instruções básicas para orientação.

O instrumento utilizado para a coleta de dados foi um questionário contendo 111 questões sobre aspectos socioeconômicos, demográficos, condições de trabalho (carga horária de trabalho semanal, turnos de trabalho, movimentos repetitivos, vibração, posição viciosa), além de comportamentais e de saúde (autopercepção de saúde, hábitos de fumar, nível de atividade física, índice de massa corporal (IMC), razão cintura-quadril (RCQ), transtornos psiquiátricos menores e problemas musculoesqueléticos), sendo as questões referentes à cor da pele, peso e altura todas autoreferidas.

As questões referentes às condições de trabalho dos motoristas e cobradores foram baseadas em diferentes questionários utilizados em estudos de avaliação desse tema em trabalhadores do transporte coletivo e trabalhadores em geral ${ }^{6}$.

O IMC dos indivíduos foi calculado pelo peso $(\mathrm{Kg})$ referido, dividido pela altura $(\mathrm{cm})$ referida elevada ao quadrado, conforme recomendações da WHO ${ }^{7}$. As medidas perímetro da cintura e do quadril foram realizadas com fita métrica inextensível (milímetros). Para medir o perímetro da cintura, a fita métrica foi posicionada na menor curvatura localizada entre as costelas e a crista ilíaca. Para aferição do perímetro do quadril, a fita métrica foi posicionada na área de maior protuberância glútea. Para avaliação do risco de complicações metabólicas segundo a $R C Q$, utilizaram-se como pontos de corte os valores de $\geq 0,90$ para homens e de $\geq 0,85$ para mulheres ${ }^{8}$.

O instrumento utilizado para avaliar o nível de atividade física nos domínios de lazer e deslocamento foi o Questionário Internacional de Atividade Física (IPAQ), versão longa. Para o presente estudo foi considerado o total de atividade nos domínios do lazer e deslocamento separadamente e em conjunto, durante uma semana habitual. Os indivíduos que relataram a prática semanal igual ou superior a 150 minutos foram considerados ativos. Para construção deste escore, o tempo gasto com a prática de caminhada, atividades físicas moderadas e vigorosas foram somados, sendo que o tempo das atividades vigorosas foi multiplicado por dois. Esta classificação vai ao encontro das recomendações atuais de atividade física ${ }^{9}$.

Os transtornos psiquiátricos menores foram identificados por meio do Self-Report Questionnaire (SRQ-20). O SRQé recomendado pela Organização Mundial de Saúde para identificar doenças psíquicas comuns e foi validado no Brasil por Mari 
e Willians ${ }^{10}$. Segundo os mesmos autores, a pontuação igual ou maior que 6 para homens e 8 ou superior para mulheres é utilizada como ponto de corte para indicativo de transtornos psiquiátricos menores ${ }^{\mathbf{1 0}}$.

Para investigar as questões relativas aos sintomas musculoesqueléticos foi utilizado o Questionário Nórdico de Sintomas Musculoesqueléticos. Esse instrumento foi adaptado culturalmente para a língua portuguesa por Barros e Alexandre em 2003 e vem sendo utilizado em estudos epidemiológicos ${ }^{11}$. O questionário coleta informações sobre dor em nove regiões anatômicas (pescoço, ombros, cotovelos, pulsos /mãos, costas, coxas, pernas, joelhos e tornozelos) usando um diagrama do corpo humano. É solicitado ao entrevistado identificar o(s) local(is) do corpo em que sentiu dor nos últimos doze meses e na última semana.

O hábito de fumar foi investigado por intermédio do instrumento proposto pelo Centro Brasileiro Sobre Drogas Psicotrópicas (CEBRID) e a realização de exame glicêmico através da pergunta "Você realizou exame de açúcar no último ano?".

Para uma amostra aleatória de 10\% dos motoristas e cobradores já entrevistados foi aplicada uma versão resumida do questionário incluindo questões essenciais do instrumento para fins de controle de qualidade e veracidade dos dados.

O banco de dados foi construído no programa Excel 2010 for Windows. Para a análise utilizou-se o programa STATA 10.0. Realizou-se uma análise descritiva dos dados com cálculo de frequência para variáveis categóricas e média, desvio-padrão e mediana para as variáveis contínuas. O teste de qui-quadrado de Pearson foi utilizado para a verificação da associação entre categoria de trabalho e inatividade física.

O Protocolo do estudo foi aprovado pelo Comitê de Ética em Pesquisas da Universidade Federal de Pelotas (Número do protocolo 007/2011) e os motoristas e cobradores assinaram um termo de consentimento livre e esclarecido antes da coleta dos dados.

\section{RESULTADOS}

Do total de 240 trabalhadores amostrados para participarem do estudo, 225 foram entrevistados (6,3\% de recusas). Todos entrevistados eram do sexo masculino e a média de idade foi de 36,2 anos ( $d p=11,6$ anos). Observou-se que 77,3\% dos entrevistados eram de cor branca, $64,9 \%$ eram casados/viviam com companheira e $69,8 \%$ tinham filhos. Em relação à escolaridade, a média de anos de estudos encontrada foi de 9,2 anos ( $d p=2,5$ anos), sendo que $16,4 \%$ possuíam ensino fundamental completo, $42,7 \%$ ensino médio completo e $4,9 \%$ nível superior completo (Tabela 1). Quanto à renda mensal, os motoristas recebiam salário de $\mathrm{R} \$ 1738,15$ e os cobradores de $\mathrm{R} \$ 1161,97$.

A tabela 2 descreve as variáveis comportamentais e de saúde dos motoristas e cobradores do transporte coletivo urbano da cidade de Pelotas/RS. Quanto à autopercepção de saúde, $14,4 \%$ dos entrevistados relatou sua saúde como regular/ ruim e mais de $21,8 \%$ dos entrevistados admitiram fumar atualmente.

A mediana de prática semanal de atividade física (atividade física nos domínios de lazer e deslocamento) foi de 252,5 minutos (média=424,3; dp=474,3), sendo que $37,5 \%$ não atingiram o mínimo de atividade física recomendada para promoção da saúde (150 min. por semana). Quando analisada somente a prática de atividade física $(\mathrm{AF})$ no domínio de lazer, quase a metade dos entrevistados $(47,1 \%)$ 
Tabela 1 - Características sociodemográficas e econômicas dos trabalhadores do transporte coletivo urbano da cidade de Pelotas/ RS, 2011. $(n=225)$

\begin{tabular}{|c|c|c|c|c|c|c|}
\hline \multirow[t]{2}{*}{ Variáveis } & \multicolumn{2}{|c|}{ Todos } & \multicolumn{2}{|c|}{ Motoristas } & \multicolumn{2}{|c|}{ Cobradores } \\
\hline & $\mathrm{n}$ & $\%$ & $\mathrm{n}$ & $\%$ & $n$ & $\%$ \\
\hline \multicolumn{7}{|l|}{ Idade (anos) } \\
\hline $17-29$ & 71 & 31,6 & 14 & 13,1 & 57 & 48,3 \\
\hline $30-39$ & 70 & 31,1 & 44 & 41,1 & 26 & 22,0 \\
\hline $40-49$ & 48 & 21,3 & 28 & 26,2 & 20 & 17,0 \\
\hline 50 ou mais & 36 & 16,0 & 21 & 19,6 & 15 & 12,7 \\
\hline \multicolumn{7}{|l|}{ Cor da pele } \\
\hline Branca & 174 & 77,3 & 86 & 80,4 & 88 & 74,6 \\
\hline Não branca & 51 & 22,7 & 21 & 19,6 & 30 & 25,4 \\
\hline \multicolumn{7}{|l|}{ Estado civil } \\
\hline Casado/vive companheiro & 146 & 64,9 & 86 & 80,4 & 60 & 50,9 \\
\hline Solteiro & 79 & 35,1 & 21 & 19,6 & 58 & 49,1 \\
\hline \multicolumn{7}{|l|}{ Escolaridade } \\
\hline Fundamental incompleto & 49 & 21,8 & 29 & 27,1 & 20 & 17,0 \\
\hline Fundamental completo & 37 & 16,4 & 24 & 22,4 & 13 & 11,0 \\
\hline Médio incompleto & 32 & 14,2 & 16 & 15,0 & 16 & 13,6 \\
\hline Médio completo & 96 & 42,7 & 34 & 31,8 & 62 & 52,5 \\
\hline Superior & 11 & 4,9 & 4 & 3,7 & 7 & 5,9 \\
\hline
\end{tabular}

não atingiu a quantidade mínima de prática de atividade física recomendada pelos órgãos de saúde, sendo que no domínio deslocamento esse valor é ainda superior $(72,3 \%)$. Em ambos os domínios da atividade física, os motoristas mostraram frequências superiores de inatividade física do que seus companheiros de trabalho ( $\mathrm{p}=$ 0,002 e $\mathrm{p}=0,01$ respectivamente para $\mathrm{AF}$ no lazer e $\mathrm{AF}$ no deslocamento).

A média de peso dos trabalhadores foi de $82,5 \mathrm{Kg}(\mathrm{dp}=12,4 \mathrm{Kg})$, sendo que a média do IMC foi $27,0 \mathrm{Kg} / \mathrm{m}^{2}\left(\mathrm{dp}=3,8 \mathrm{Kg} / \mathrm{m}^{2}\right)$ e apresentaram risco aumentado para complicações metabólicas segundo a relação cintura quadril, 57,5\% dos indivíduos avaliados.

De acordo com a classificação do índice de massa corporal (IMC), nas categorias referentes a sobrepeso e obesidade, o percentual relativo a estas duas categorias somadas atingiu quase $3 / 4 \mathrm{da}$ amostra.

Em relação à saúde mental, a prevalência de transtornos psiquiátricos menores foi de $8,6 \%$, sendo, em torno de $6,6 \%$ entre os motoristas e de $10,4 \%$ entre os cobradores. No que se refere à presença de sintomas musculoesqueléticos no último ano, verificou-se que a maioria dos relatos referia a desconforto e/ou dor nas regiões da coluna lombar $(48,4 \%)$, coluna torácica $(39,4 \%)$, ombros $(38,5 \%)$ e pescoço (36,6\%). Quando analisados separadamente por categoria, a dor lombar é a mais frequente para ambas mas, entre os cobradores, a dor no pescoço supera a dor nos ombros. Entre os que relataram dor lombar no último ano, 32,4\% afirmaram que esse problema o impediu de realizar tarefas domésticas ou de trabalho. A média de regiões relatadas com dor no período do último ano foi de 2,5 (dp = 2,4). Quanto as análises bioquímicas, mais de $2 / 3(71 \%)$ realizaram exame para verificação da glicemia no último ano (Tabela 2), sendo que, dos que realizaram, 9,7\% apresentaram valores de glicemia igual ou superior a $140 \mathrm{mg} / \mathrm{dL}$. 
Tabela 2 - Frequência absoluta e relativa dos trabalhadores do transporte coletivo urbano segundo variáveis comportamentais e de saúde, Pelotas/RS, 2011. $(n=225)$.

\begin{tabular}{|c|c|c|c|c|c|c|}
\hline \multirow{2}{*}{ Variáveis } & \multicolumn{2}{|c|}{ Total } & \multicolumn{2}{|c|}{ Motoristas } & \multicolumn{2}{|c|}{ Cobradores } \\
\hline & $\mathrm{n}$ & $\%$ & $n$ & $\%$ & $n$ & $\%$ \\
\hline \multicolumn{7}{|l|}{ Percepção de saúde } \\
\hline Excelente & 38 & 17,1 & 14 & 13,1 & 24 & 20,9 \\
\hline Muito boa & 32 & 14,4 & 14 & 13,1 & 18 & 15,6 \\
\hline Boa & 120 & 54,1 & 63 & 58,9 & 57 & 49,6 \\
\hline Regular/ruim & 32 & 14,4 & 16 & 14,9 & 16 & 13,9 \\
\hline \multicolumn{7}{|l|}{ Hábito de fumo atual } \\
\hline Não & 176 & 78,2 & 86 & 80,4 & 90 & 76,3 \\
\hline Sim & 49 & 21,8 & 21 & 19,6 & 28 & 23,7 \\
\hline \multicolumn{7}{|l|}{ Atividade física no lazer } \\
\hline Insuficientemente ativo & 106 & 47,1 & 62 & 57,9 & 44 & 37,3 \\
\hline Ativo & 119 & 52,9 & 45 & 42,1 & 74 & 62,7 \\
\hline \multicolumn{7}{|c|}{ Atividade física no deslocamento } \\
\hline Insuficientemente ativo & 162 & 72,3 & 86 & 80,4 & 76 & 65,0 \\
\hline Ativo & 62 & 27,7 & 21 & 19,6 & 41 & 35,0 \\
\hline \multicolumn{7}{|c|}{ Atividade física lazer+deslocamento } \\
\hline Insuficientemente ativo & 84 & 37,5 & 54 & 50,5 & 30 & 25,6 \\
\hline Ativo & 140 & 62,5 & 53 & 49,5 & 87 & 74,4 \\
\hline \multicolumn{7}{|c|}{ Índice de massa corporal (IMC) } \\
\hline Normal & 65 & 29,0 & 27 & 25,5 & 38 & 32,2 \\
\hline Sobrepeso & 108 & 48,2 & 55 & 51,9 & 53 & 44,9 \\
\hline Obeso & 51 & 22,8 & 24 & 22,6 & 27 & 22,9 \\
\hline \multicolumn{7}{|c|}{ Razão cintura quadril (RCQ)\# } \\
\hline Risco & 126 & 57,5 & 73 & 68,9 & 53 & 46,9 \\
\hline Não risco & 93 & 42,5 & 33 & 31,1 & 60 & 53,1 \\
\hline \multicolumn{7}{|c|}{ Transtornos psiquiátricos menores } \\
\hline Não & 202 & 91,4 & 99 & 93,4 & 103 & 89,6 \\
\hline Sim & 19 & 8,6 & 7 & 6,6 & 12 & 10,4 \\
\hline \multicolumn{7}{|c|}{ Positividade de sintomas musculoesqueléticos no último ano* } \\
\hline Dor pescoço & 81 & 36,6 & 47 & 44,3 & 34 & 29,6 \\
\hline Dor ombros & 85 & 38,5 & 54 & 50,9 & 31 & 27,0 \\
\hline Dor cotovelos & 14 & 6,3 & 4 & 3,8 & 10 & 8,7 \\
\hline Dor pulsos e mãos & 27 & 12,2 & 13 & 12,3 & 14 & 12,2 \\
\hline Dor coluna torácica & 87 & 39,4 & 47 & 44,3 & 40 & 34,8 \\
\hline Dor coluna lombar & 107 & 48,4 & 63 & 59,4 & 44 & 38,2 \\
\hline Dor coxas & 24 & 10,9 & 8 & 7,6 & 16 & 13,9 \\
\hline Dor pernas & 47 & 21,3 & 24 & 22,6 & 23 & 20,0 \\
\hline Dor joelhos & 52 & 23,5 & 29 & 27,4 & 23 & 20,0 \\
\hline Dor tornozelos & 18 & 8,1 & 13 & 12,3 & 5 & 4,4 \\
\hline \multicolumn{7}{|l|}{ Glicemia medida último ano } \\
\hline Sim & 157 & 71,0 & 84 & 79,3 & 73 & 63,5 \\
\hline Não & 64 & 29,0 & 22 & 20,7 & 42 & 36,5 \\
\hline
\end{tabular}

* O somatório das frequências (\%) de dor não são iguais a 100\% * Variável com maior número de missings $(n=6)$.

Em média estes indivíduos pertenciam ao quadro funcional das empresas de transporte coletivo urbano da cidade de Pelotas/RS há 8,9 anos ( $\mathrm{dp}=6,8$ anos), 
com carga horária diária média de 7,1 h $(\mathrm{dp}=0,7 \mathrm{~h})$. Apenas $1 / 4$ do total de entrevistados também já trabalhou em outra empresa nesta atual função (Tabela 3).

Tabela 3 - Frequência absoluta e relativa dos trabalhadores do transporte coletivo urbano da cidade de Pelotas/ RS segundo variáveis ocupacionais, Pelotas, 2011. ( $n=225)$

\begin{tabular}{|c|c|c|c|c|c|c|}
\hline \multirow[t]{2}{*}{ Variáveis } & \multicolumn{2}{|c|}{ Todos } & \multicolumn{2}{|c|}{ Motoristas } & \multicolumn{2}{|c|}{ Cobradores } \\
\hline & $\mathrm{n}$ & $\%$ & $\mathrm{n}$ & $\%$ & $\mathrm{~N}$ & $\%$ \\
\hline \multicolumn{7}{|c|}{ Tempo de trabalho na função } \\
\hline Menos de 1 ano & 17 & 7,6 & 4 & 3,7 & 13 & 11,0 \\
\hline Um a 5 anos & 92 & 40,9 & 35 & 32,7 & 57 & 48,3 \\
\hline 5,1 a 10 anos & 30 & 13,3 & 24 & 22,4 & 6 & 5,1 \\
\hline 10,1 a 15 anos & 34 & 15,1 & 15 & 14,0 & 19 & 16,1 \\
\hline 15,1 a 20 anos & 19 & 8,4 & 7 & 6,5 & 12 & 10,2 \\
\hline 20,1 ou mais anos & 33 & 14,7 & 22 & 20,7 & 11 & 9,3 \\
\hline \multicolumn{7}{|l|}{ Satisfação no trabalho } \\
\hline Sim & 206 & 92,8 & 97 & 90,6 & 109 & 94,8 \\
\hline Não & 16 & 7,2 & 10 & 9,4 & 6 & 5,2 \\
\hline \multicolumn{7}{|c|}{ Desejam mudar a função atual que exercem na empresa } \\
\hline Sim & 68 & 30,6 & 1 & 0,9 & 67 & 58,2 \\
\hline Não & 154 & 69,4 & 106 & 99,1 & 48 & 41,8 \\
\hline \multicolumn{7}{|c|}{ Realizam movimentos repetitivos } \\
\hline Sim & 195 & 88,2 & 104 & 98,1 & 91 & 79,1 \\
\hline Não & 26 & 11,8 & 2 & 1,9 & 24 & 20,9 \\
\hline \multicolumn{7}{|c|}{ Trabalham em posição estática ou viciosa } \\
\hline Sim & 207 & 92,4 & 99 & 92,5 & 108 & 92,3 \\
\hline Não & 17 & 7,6 & 8 & 7,5 & 9 & 7,7 \\
\hline \multicolumn{7}{|c|}{ Expostos a vibração de corpo todo } \\
\hline Sim & 211 & 94,2 & 101 & 94,4 & 110 & 94,0 \\
\hline Não & 13 & 5,8 & 6 & 5,6 & 7 & 6,0 \\
\hline \multicolumn{7}{|c|}{$\begin{array}{l}\text { Trafegam por ruas pavimen- } \\
\text { tadas }\end{array}$} \\
\hline Sim & 100 & 45,0 & 53 & 50,5 & 47 & 40,2 \\
\hline Não & 122 & 55,0 & 52 & 49,5 & 70 & 59,8 \\
\hline \multicolumn{7}{|l|}{ Sofreram assalto } \\
\hline Sim & 117 & 52,7 & 60 & 56,1 & 57 & 50,4 \\
\hline Não & 105 & 47,3 & 47 & 43,9 & 58 & 49,6 \\
\hline
\end{tabular}

Entre os profissionais estudados, $88,2 \%$ responderam realizar movimentos repetitivos durante o turno de trabalho, $92,4 \%$ relataram permanecer em posições viciosas / incômodas frequentemente, sendo a posição sentada a mais citada $(99,6 \%)$. O tempo médio para cada volta dos itinerários foi de 58,6 minutos (dp $=16,8)$, sendo que $94,2 \%$ sofrem vibrações e mais da metade trafega por ruas não pavimentadas. Quanto a estar satisfeito com sua atividade profissional, mais de $92,8 \%$ respondeu positivamente, porém mais da metade relatou ter sido assaltado durante o trabalho (Tabela 3).

\section{DISCUSSÃO}

Os achados do estudo descrevem as condições de saúde, trabalho e indicadores do estilo de vida de trabalhadores do transporte público urbano de uma cidade de 
porte médio do Brasil. Apontam resultados importantes tais como as prevalências preocupantes de sobrepeso/obesidade, inatividade física, dor musculoesquelética, movimentos repetitivos e posição estática ou viciosa no trabalho, as quais podem influenciar negativamente no desempenho no trabalho e na saúde desses indivíduos.

No presente estudo a média de idade encontrada foi semelhante à de outros estudos com trabalhadores do transporte coletivo ${ }^{1,12}$. Quando esta variável foi analisada de forma categorizada para motoristas e cobradores separadamente, verificou-se uma maior prevalência de cobradores entre 17 e 29 anos e de motoristas entre 30 e 39 anos, distribuição semelhante à encontrada nos estudos de Faria et al. ${ }^{13}$ e Costa et al. ${ }^{14}$. Isso pode estar relacionado ao fato de que somente indivíduos com idade mínima de 21 anos e possuindo pelo menos dois anos de habilitação na categoria $\mathrm{B}$ ou, no mínimo, um ano na categoria $\mathrm{C}$ podem prestar exame para habilitação na categoria compatível ao transporte de pessoas (categoria D).

O sexo masculino representa quase a totalidade dos trabalhadores dessas profissões no município de Pelotas, o que é semelhante em outros estudos ${ }^{1,6,16}$. Segundo Guterres e colaboradores ${ }^{16}$, o trabalho de cobrador e motorista é uma jurisdição dominada por trabalhadores do sexo masculino. Esse comportamento parece estar relacionado à periculosidade da profissão, considerando o elevado número de assaltos e acidentes de trânsito ${ }^{17}$.

A categoria de escolaridade com maior percentual de trabalhadores no presente estudo foi a do ensino médio completo, dado também encontrado por Costa et al. ${ }^{14}$. Tais achados divergem dos de Moraes onde apenas 3\% da amostra completaram o ensino médio ${ }^{18}$. Tal diferença encontrada entre os estudos pode estar relacionada ao nível de exigência educacional no processo de admissão em diferentes épocas, já que os mesmos acontecem em intervalo de quase dez anos. A aparente estabilidade profissional e a não exigência de grau elevado de estudo para admissão, aliado a pouca ou nenhuma perspectiva de promoção nas empresas pode desestimular a formação educacional institucionalizada.

Mais da metade dos entrevistados trabalha nas empresas há pelo menos cinco anos. Estudo de Bevengnú e colaboradores ${ }^{19}$ realizado em Santa Maria, cidade de porte médio no mesmo estado do presente estudo, mostrou a variável tempo de trabalho, em anos, semelhante. Entretanto, Rezende ${ }^{20}$, em estudo realizado em trabalhadores do transporte coletivo da cidade de Belo Horizonte, encontrou tempo de trabalho inferior aos do presente estudo, fato que pode estar relacionado com o tamanho da cidade. O tamanho da cidade é um indicativo de maior oferta e precarização de empregos, o que pode favorecer a mobilidade dos trabalhadores para outros serviços. Apesar dessa hipótese, o tempo de trabalho elevado encontrado no presente estudo pode ser suportado pela satisfação e bem estar na atividade laboral que realiza (mais de $90 \%$ dos trabalhadores relataram satisfação com a atividade laboral), além da relativa estabilidade no serviço com carteira assinada. Apesar disso, 58,2\% dos cobradores relataram o desejo de mudar para função de motorista, comportamento possivelmente influenciado pelo maior salário e status social dos motoristas.

A frequência de fumantes atuais $(21,8 \%)$ é semelhante à encontrada em estudos com motoristas e cobradores no país ${ }^{14,21}$. Apesar de essa prevalência ser inferior a encontrada em trabalhadores do transporte na Austrália $(36,9 \%)^{22}$ e de várias categorias ocupacionais nos Estados Unidos $(>30,0 \%)^{23}$, ainda são muito superioras as encontradas em profissionais com maior qualificação no Brasil $\left(9,4 \%{ }^{24}\right.$. Isso pode estar relacionado com os menores níveis educacionais dessa 
categoria ${ }^{24}$ e pelo hábito de fumar entre os homens no Rio Grande do Sul ser um dos maiores do país ${ }^{25}$

No presente estudo quase 3/4 (71,0\%) dos indivíduos foram classificados nas categorias de sobrepeso e obesidade segundo o IMC e a prevalência encontrada referente a risco aumentado para complicações metabólicas foi de 57,5\% segundo a relação cintura/quadril, valor este superior ao encontrado em outro estudo com motoristas ${ }^{14}$. Os resultados para o IMC foram semelhantes e reforçados pelos encontrados em outros estudos ${ }^{15,18}$ e que também suportam os dados descritos por Guterres et al. ${ }^{16}$, em pesquisa realizada na mesma cidade em anos anteriores. Faria et al. ${ }^{13}$ descreve em seu estudo que $67,3 \%$ dos entrevistados encontravam-se nas categorias de sobrepeso/obesidade, enquanto que, na época de admissão na profissão, essa prevalência era de 30,9\%. A longa jornada de trabalho, realizada em turnos e sedentária (trabalho sentado), e estilo de vida inativo são fatores contribuintes para o aumento de peso ${ }^{5}$.

A prática da atividade física é fator reconhecidamente importante para prevenção e tratamento de doenças crônicas na população. Entre trabalhadores do transporte coletivo urbano, a mesma tem sua contribuição no sentido de minimizar os impactos da atividade laboral sedentária, as quais conduzem direta e indiretamente ao risco aumentado de doenças. A importância desse tema em trabalhadores data de 1953 quando Morris et al. ${ }^{26}$ estudaram o risco de ser inativo para a mortalidade por doenças cardiovasculares nessa população. $O$ presente estudo diagnosticou prevalências elevadas de inatividade física tanto nos domínios de lazer e deslocamento separadamente, como conjuntamente. Resultados de estudos com motoristas e cobradores mostram prevalências elevadas de inatividade física, variando de $64,5 \%{ }^{6}$ a $76,0 \%{ }^{19}$ independentemente do instrumento utilizado para a coleta de dados. Tal achado permite sugerir que sejam adotadas medidas no sentido de indicar, orientar e oportunizar a prática de alguma atividade física ou esporte regularmente, visando melhorar as condições de saúde dos trabalhadores.

As prevalências de algumas doenças crônicas não transmissíveis encontradas no estudo são preocupantes. Exemplo disso foram as prevalências de dor encontradas para as regiões dos ombros, lombar e pescoço durante o último ano. Relatos semelhantes vêm sendo descritos em outros estudos com esses profissionais ${ }^{16,18}$. Segundo Battiston ${ }^{1}$, o mau estado de conservação e a deficitária ergonomia dos veículos, tais como, falta de direção e assento ajustáveis, falta de apoio anatômico para as costas, a não existência de cinto de segurança de três pontos e localização do motor na parte traseira do ônibus são fatores comumente encontrados nos coletivos municipais que podem estar associados ao surgimento e/ou agravamento da dor. Guterres et al. ${ }^{16}$ acrescentam que, fatores externos ao ônibus, como as precárias condições das vias de trânsito e a ausência de corredores de coletivos na cidade podem ajudar a aumentar o número de indivíduos com dor, sendo que no atual estudo mais da metade dos trabalhadores relataram trafegar por vias sem pavimentação. Alguns autores relatam ainda, que o elevado tempo de permanência na posição sentada ${ }^{27}$, aliado ao excesso de peso ${ }^{28}$ pode contribuir para o acometimento de dores na região lombar, corroborando com os presentes achados onde mais de $90 \%$ dos entrevistados afirmaram trabalhar em uma mesma posição a maior parte do tempo além de sofrerem com vibrações no corpo inteiro.

No presente estudo quase a totalidade dos motoristas e mais de $3 / 4$ dos cobradores relataram realizar movimentos repetitivos. Queiroga e Michels ${ }^{12}$ afirmam que as frequentes rotações da cabeça e do tronco afetam a coluna vertebral e que 
as trocas constantes de marcha podem resultar em bursite ou tendinite nos ombros. Santos Filho e Barreto ${ }^{29}$ descrevem que a dor osteomuscular pode provocar sérias consequências sociais, como limitações no trabalho e índice de absenteísmo e afastamentos, e execução de atividades da vida diária.

Landim e Victor ${ }^{21}$ detectaram baixa prevalência de motoristas com diabete melitus $(3,7 \%)$, porém os autores salientam a necessidade de controle da glicemia pelas consequências que o desenvolvimento deste distúrbio pode trazer aos trabalhadores. Neste estudo mais de 70\% realizaram exames de glicemia, fator este positivo para controle deste acometimento. A prevalência inferior a $10,0 \%$ de indivíduos com glicemia alterada pode estar relacionada ao viés do trabalhador sadio na medida em que as complicações da glicemia elevada podem prejudicar e afastar os indivíduos de suas funções laborais. É importante salientar que, mais do que uma necessidade, a realização de exames físicos e psicológicos periódicos são uma obrigatoriedade legal por parte do empregador, não só porque da saúde do trabalhador depende a segurança de muitas pessoas, como também porque inúmeras conquistas trabalhistas dependem da avaliação periódica das condições de saúde e trabalho dos profissionais, independente da categoria ocupacional.

Motoristas e cobradores de ônibus defrontam-se diariamente com uma série de fatores ocupacionais que podem estar relacionados ao surgimento de transtornos psiquiátricos menores, especialmente depressão e ansiedade. Estudo de revisão sistemática sobre transtornos mentais indica que a rotina de trabalho, a demanda, a exigência, o controle, o processo de trabalho, as condições ambientais estão associados ao surgimento desses distúrbios ${ }^{30}$. O presente estudo diagnosticou prevalências indicativas de TPM em torno de 10\%, semelhante à encontrada por Bevengnú em Santa Maria/RS ${ }^{19}$ e inferior a encontrada por Souza e Silva em São Paulo ${ }^{31}$. Os cobradores parecem ser mais vulneráveis, pois exercem um importante papel na organização e acomodação dos passageiros no ônibus, administrando o espaço e auxiliando de diversas formas o motorista, sendo que a relação com os passageiros encontra-se no núcleo de seu processo de trabalho, tornando-os mais expostos. Nosso estudo identificou que mais de 50\% dos cobradores já haviam sido assaltados no trabalho o qual é fator que pode estar relacionado ao aparecimento de TPM.

O estudo analisou uma amostra representativa dos profissionais do transporte urbano do município de Pelotas e, portanto, a extrapolação de alguns dados encontrados para motoristas e cobradores de outras cidades deve ser avaliada com cautela. Apesar de muitas características da atividade profissional desses trabalhadores serem semelhantes, independentemente do tamanho da cidade onde o trabalho aconteça, outros fatores apresentam-se diferenciados, como a quantidade de carros no trânsito, a pavimentação e a largura das ruas e a violência nos municípios, entre outros, o que pode gerar resultados divergentes sobre o mesmo assunto. Outro ponto a ser salientado é o fato de que a listagem fornecida pelas empresas somente continha o nome dos trabalhadores ativos. Isso nos impossibilitou saber quem foram os indivíduos afastados ou demitidos do serviço por problema de saúde, o que poderia elevar a prevalência de algumas variáveis relacionadas ao tema.

Concluindo, a prevalência de indicadores da má condição do estilo de vida, da saúde e do trabalho dos funcionários das empresas do transporte coletivo urbano da cidade de Pelotas foi elevada. Entre elas, destacam-se o sobrepeso/obesidade, a inatividade física, as dores/desconfortos musculoesqueléticos nas regiões lombar, torácica, ombro e pescoço, os movimentos repetitivos e posições forçadas no trabalho. Apesar de não tão prevalente, a frequência de TPM entre os trabalhadores 
também é importante, pois expõe os mesmos e, consequentemente, os usuários a maior risco de acidentes no trânsito. $\mathrm{O}$ diagnóstico das condições de trabalho e saúde dos motoristas e cobradores deve servir de subsídio às empresas para a implantação de programas de hábitos de vida saudável, como deslocamento ativo, prática de atividades físicas regulares, bem como a melhoria das condições de trabalho como adequação das pausas de trabalho, assim como a melhoria das condições de trafegabilidade e segurança por parte dos órgãos públicos.

\section{Agradecimentos}

Os autores deste artigo agradecem às empresas de transporte coletivo urbano da cidade de Pelotas/RS, pela coleta de dados.

\section{REFERÊNCIAS}

1. Battiston M, Cruz R, Hoffmann MH. Condições de trabalho e saúde de motoristas de transporte coletivo urbano. Estud Psicol 2006;11:333-43.

2. Costa EAVG. Estudos dos constrangimentos físicos e mentais sofrido pelos motoristas de ônibus urbano da cidade do Rio de Janeiro. Dissertação de Mestrado, PUC, Rio de Janeiro, Rio de Janeiro, 2006.

3. Instituto de Pesquisa Econômica Aplicada (Ipea). Sistema de Indicadores de Percepção Social: Mobilidade Urbana. Acessado em 10 de Março de 2012. Disponível em: http://www.ipea.gov. br/portal/images/stories/PDFs/SIPS/110504_sips_mobilidadeurbana.pdf

4. Picoloto D, Silveira E. Prevalência de sintomas osteomusculares e fatores associados em trabalhadores de uma indústria metalúrgica de Canoas - RS. Ciênc Saúde Coletiva 2008;13:507-16.

5. Winkleby MA, Ragland DR, Fisher JM et al. Excess risk of sickness and disease in bus drivers: A review and synthesis of epidemiologic studies. Int J Epidemiol 1988;17:255-262.

6. Deus MJ. Comportamento de Risco à Saúde e Estilo de Vida em Motoristas de Ônibus Urbanos: recomendações para um programa de promoção de saúde. Tese de Doutorado, UFSC, Florianópolis, Santa Catarina, 2005.

7. World Health Organization. Physical Status: The Use and Interpretation of Anthropometry. Geneva: World Health Organization; 1995.

8. World Health Organization. Waist circumference and waist-hip ratio: report of a WHO expert consultation. 2008. Acessado em: 20 de Março de 2012. Disponível em: http://whqlibdoc. who.int/publications/2011/9789241501491_eng.pdf.

9. Haskell WL, Lee I, Pate RR et al. Physical Activity and Public Health: Updated Recommendation for Adults From the American College of Sports Medicine and the American Heart Association. Circulation 2007; 116:1081-93.

10. Mari J, Williams PA. Validity study of a Psychiatric Screening Questionnaire (SRQ-20) in Primary Care in the city of São Paulo. Br J Psychiatry 1986;148:23-6.

11. Barros ENC, Alexandre NMC. Cross-cultural adaptation of the Nordic musculoskeletal questionnaire. Int Nurs Ver 2003;50:101-08.

12. Queiróga MR, Michels G. A influência de características individuais na incidência de dor músculo-esquelética em motoristas de ônibus da cidade de Londrina. Rev Bras Ativ Fis e Saúde 1999;2:49-61.

13. Faria BK, Amorim G, Vancea DMM. Perfil alimentar e antropométrico dos motoristas de ônibus da empresa de transporte coletivo Jotur/Palhoça-SC. Rev Brasil Obesid Nut Emag 2007;1:11-20.

14. Costa MM, Mastroeni SSBS, Reis MAM et al. Excesso de peso em motoristas de ônibus da rede urbana. R Bras Ci e Mov 2011;19:42-51.

15. Costa LB, Koyama MH, Minuci EG et al. Morbidade declarada e condições de trabalho: o caso dos motoristas de São Paulo e Belo Horizonte. São Paulo Perspec 2003;17.

16. Guterres A, Duarte D, Siqueira FV et al. Prevalência e fatores associados a dor nas costas dos motoristas e cobradores do transporte coletivo da cidade de Pelotas-RS. Rev Bras Ativ Fis e Saúde 2011;16:240-45. 
17. Mendes LR. Condições de trabalho no transporte coletivo: desgaste e responsabilidade do motorista de ônibus. In: Sampaio J R, Organizador. Qualidade de vida, saúde mental e psicologia social. São Paulo: Casa do psicólogo 1999: 153-80.

18. Moraes LFS. Os princípios das cadeias musculares na avaliação dos desconfortos corporais e constrangimentos posturais em motoristas do transporte coletivo. Dissertação de Mestrado, UFSC, Florianópolis, Santa Catarina, 2002.

19. Benvegnú L, Fassa AG, Facchini LA et al. Prevalência de hipertensão arterial entre motoristas de ônibus em Santa Maria, Rio Grande do Sul. Rev Bras Saúde Ocup 2008; 33:32-39.

20. Rezende AM. Situações de trabalho e riscos ocupacionais: um estudo de caso com os motoristas de transporte urbano por ônibus. Dissertação de Mestrado, UFMG, Belo Horizonte, Minas Gerais, 2001.

21. Landim MBP, Victor EG. Framingham Score for Public Transportation Drivers in the City of Teresina, Piauí. Arq Bras Cardiol 2006; 87: 315-20.

22. Smith DR, Leggat PA. Tobacco smoking by occupation in Australia: results from the 2004 to 2005 National Health Survey. J Occup Environ Med 2007; 49:437-445.

23. Lee DJ, Fleming LE, Arheart KL et al. Smoking rate trends in U.S. occupational groups: the 1987 to 2004 National Health Interview Survey. J Occup Environ Med 2007; 49:75-81.

24. Barros AJ, Cascaes AM, Wehrmeister FC et al. Tabagismo no Brasil: desigualdades regionais e prevalência segundo características ocupacionais. Ciênc Saúde Coletiva 2011;16:3707-16.

25. Brasil. VIGITEL Brasil 2010: Vigilância de fatores de risco e proteção para doenças crônicas por inquérito telefônico. Brasília: Ministério da Saúde; 2011.

26. Morris JN, Heady JA, Raffle PAB et al. Coronary heart disease and physical activity of work. Lancet 1953; 265: 1053-57.

27. Santos CM. Enfoque ergonômico dos postos de trabalho. Revista CIPA, p.18-28, 1998.

28. Nahas MV. Atividade física, saúde e qualidade de vida. Londrina: Midiograf, 2001.

29. Santos Filho SB, Barreto SM. Atividade ocupacional e prevalência de dor osteomuscular em cirurgiões-dentistas de Belo Horizonte, Minas Gerais, Brasil: contribuição ao debate sobre os distúrbios osteomusculares relacionados ao trabalho. Cad Saúde Pública 2001; 17: 181-93.

30. Santos EG, Siqueira MM. Prevalência dos transtornos mentais na população adulta brasileira: uma revisão sistemática de 1997 a 2009. J Bras Psiquiatr 2010;59:238-46.

31. Souza MFM, Silva GR. Risco de distúrbios psiquiátricos menores em área metropolitana na região Sudeste do Brasil. Rev Saúde Pública 1998;32:50-8.

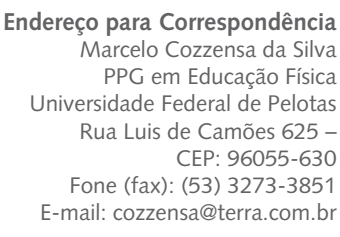

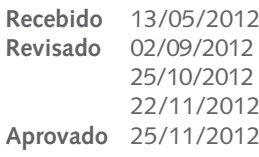

